\title{
29. In situ Obervation of the Pressure Induced Amorphization of $\mathrm{GeO}_{2}$
}

\author{
By Shinji Kawasaki, ${ }^{*)}$ Hiroshi TaKubo, ${ }^{* *)}$ and Takamitsu YamanaKA**) \\ (Communicated by Syun-iti AкIмото, м. J. A., Nov. 12, 1992)
}

\begin{abstract}
In order to study the stability field of $\mathrm{GeO}_{2}$ under high pressure at room temperature, an in situ observation was made by using a diamond anvil pressure cell, an optical microscope and a high speed VTR. At about $6.5 \mathrm{GPa}$, a transparent single-crystal of $\alpha$-quartz type $\mathrm{GeO}_{2}$ quickly changed to a translucent material which was found to be X-ray amorphous. The loss of transparency induced by the transformation is attributed to the pulverization due to the large volume change (approximately 10\%), which causes the increase of refractive index and introduces the imperfection into the single-crystal.
\end{abstract}

Key words: $\mathrm{GeO}_{2}$; amorphization; high pressure.

1. Introduction. Pressure induced amorphization was firstly investigated for $\mathrm{H}_{2} \mathrm{O} .^{1)}$ Recently, it has been found that several materials were transformed from a crystalline to an amorphous state by compression. ${ }^{2-5)}$ We reported the X-ray powder diffraction study of the pressure induced amorphization of $\mathrm{GeO}_{2} \cdot{ }^{6}$ ) The amorphization of $\mathrm{GeO}_{2}$ could be observed at about 6-9 GPa by the decrease of the diffracted intensity. However, since the diffracted intensity decreased gradually between 6 and $9 \mathrm{GPa}$, the amorphization pressure was not determined exactly. Therefore, it was difficult to discuss the kinetics and mechanism (diffusion or nondiffusion process) of the transformation. The purpose of this investigation is to elucidate dynamics of the amorphization, which includes following problems; (1) position of nucleation, (2) rate of the transformation, (3) pretransitional change of matrix phase.

There are two stable polymorphs ( $\alpha$-quartz and rutile types) of $\mathrm{GeO}_{2}$. For convenience' sake, $\alpha$-quartz type and rutile type $\mathrm{GeO}_{2}$ are abbreviated to $\mathrm{q}-\mathrm{GeO}_{2}$ and $r-\mathrm{GeO}_{2}$, respectively. The space group of $\mathrm{q}-\mathrm{GeO}_{2}$ and $\mathrm{r}-\mathrm{GeO}_{2}$ is $\mathrm{P}_{1} 21$ and $\mathrm{P} 4_{2} / \mathrm{mnm}$, respectively. The structure of $\mathrm{q}-\mathrm{GeO}_{2}$ is characterized by a $\mathrm{GeO}_{4}$ tetrahedral framework system and a germanium atom is coordinated by four oxygen atoms. On the other hand, a germanium atom in $\mathrm{r}-\mathrm{GeO}_{2}$ is surrounded by six oxygen atoms in distorted octahedral coordination.

2. Experimental. The crystal of $\mathrm{q}-\mathrm{GeO}_{2}$ was prepared by the slow-cooling technique using the powder mixture of $\mathrm{GeO}_{2}(0.01 \mathrm{~mol})$ and $\mathrm{Li}_{2} \mathrm{Mo}_{2} \mathrm{O}_{7}(0.036 \mathrm{~mol}){ }^{7}{ }^{7}$ The mixture was homogenized by keeping at $1400 \mathrm{~K}$ for 12 hours, and then cooled to $1100 \mathrm{~K}$ at a rate of 3 $\mathrm{K} /$ hour. At $1100 \mathrm{~K}$, the Pt crucible was taken out from the furnace and cooled to room temperature. The sizes of the grown crystal were in the range from a few tens micron to several millimeter. An inclusion-free transparent crystal with dimension of $0.08 \times 0.07 \times$ $0.03 \mathrm{~mm}$ was selected for the present experiment. The experimental run was carried out by using a setup incorporating with a diamond anvil pressure cell (DAC), a transmitting microscope and a high speed VTR. The DAC used was originally designed by Yamaoka et $a l .{ }^{8)}$ The culet of the opposed diamond anvils was $0.5 \mathrm{~mm}$ in diameter. The sample chamber was composed of the gasket hole with $0.1-0.2 \mathrm{~mm}$ in diameter. The gasket was stainless

*) Faculty of Science, Hokkaido University, Sapporo, Hokkaido 060, Japan.

**) College of General Education, Osaka University, Toyonaka, Osaka 560, Japan. 
steel foil which was initially $0.2 \mathrm{~mm}$ thick and preindented to a thickness of about $0.1 \mathrm{~mm}$. The mixture of methanol:ethanol=4:1 was used as a pressure transmitting medium. It was reported that the medium did not freeze up to $10 \mathrm{GPa}^{9)}$ The applied pressure was measured by the peak shift of $R_{1}$ fluorescence line of ruby. ${ }^{10)}$ The pressure was manually controlled and slowly increased with the rate less than $0.1 \mathrm{GPa} /$ second. During the run, the image of crystal under the microscope was monitored and recorded by a high speed VTR (Nac HSV-200). This VTR is able to store the image with the rate of 200 frames per second, corresponding to $5 \mathrm{~ms}$ for one frame.

3. Results and discussion. The crystal held in DAC was verified to be $q-\mathrm{GeO}_{2}$ by X-ray diffraction. As shown in Figs. 1-(1), (2), the transparent single-crystal abruptly changed to the translucent or almost opaque material at pressures around $6.5 \mathrm{GPa}$. In order to identify the translucent material thus transformed, an X-ray diffraction study was made for the sample held at nearly the same condition as stated above. As seen in Fig. 2, the X-ray powder diffraction intensity of $\mathrm{q}-\mathrm{GeO}_{2}$ started to be weakened from about $6 \mathrm{GPa}$ and the diffraction pattern disappeared completely at pressure above $9 \mathrm{GPa}^{6}{ }^{6}$ There was no evidence showing the presence of the crystalline material such as $q-\mathrm{GeO}_{2}$ or $\mathrm{r}-\mathrm{GeO}_{2}$. Therefore, the transformation can be resulted from the amorphization of $q-\mathrm{GeO}_{2}$.

The rate of transformation from $\mathrm{q}-\mathrm{GeO}_{2}$ to an amorphous substance was examined by replaying the VTR frame by frame. The transformation was completed within a time duration shorter than $5 \mathrm{~ms}$. The volume change due to the transformation was estimated from the data by an image processing system. The area of sample reduced by $5-7 \%$ through the transformation. Assuming that an isotropic shrinkage occurred, the volume change was evaluated to be $7-11 \%$. The drastic volume change was considered not to be from the elastic deformation but to the reconstructive transformation, since the compression rate did not exceed $0.1 \mathrm{GPa} /$ second.

The change of the transparency due to the amorphization can be explained as follows. The amorphization took place with the volume shrinkage of 7-11\%. If nucleation occurred at several places in the single-crystal, shear stress caused by the large volume change propagates. Thereby the single-crystal had to be polycrystallized to fine particles for the relaxation of the shear stress. The polycrystallization brought about the increase of the reflection and the decrease in the apparent transparency of the sample.
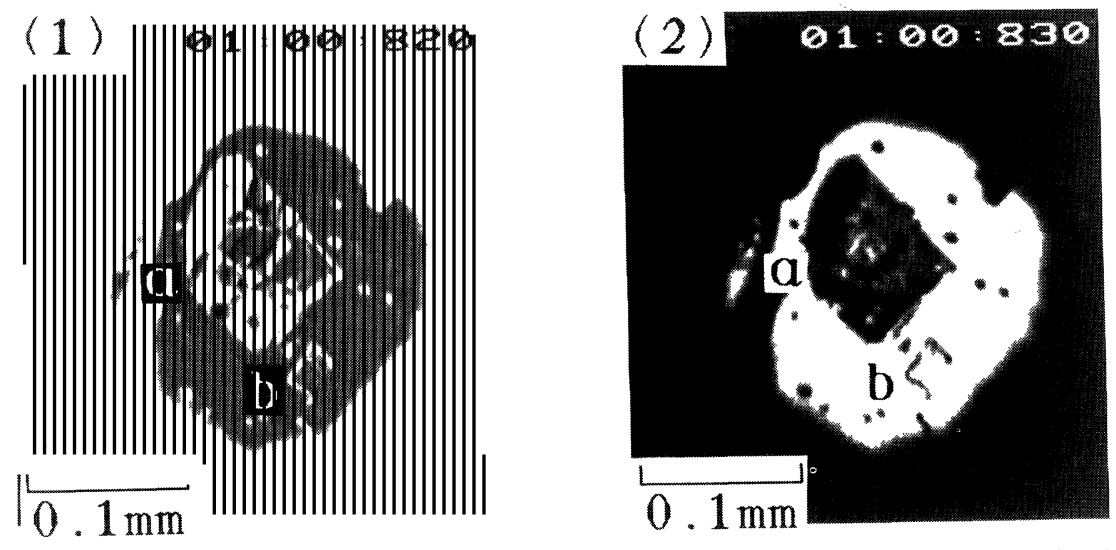

Figs. 1-(1), (2). Change of transmitting light image of $\mathrm{GeO}_{2}$ crystal by amorphization. Time duration from (1) to (2) is $10 \mathrm{~ms}$. $\mathrm{GeO}_{2}$ sample is marked a and ruby pressure marker is marked $\mathbf{b}$. Since magnification scale is the same in each image, the shrinkage of $\mathrm{GeO}_{2}$ crystal through amorphization is seen by comparing the sample area. 


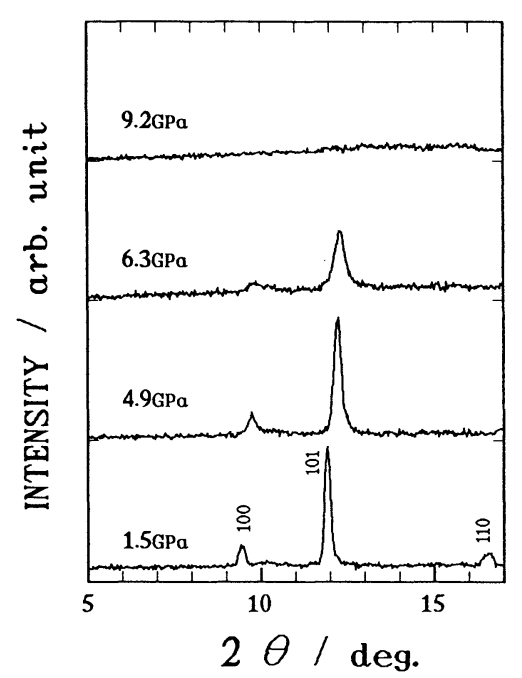

Fig. 2. Change of X-ray powder diffraction pattern of $\mathrm{q}-\mathrm{GeO}_{2}$ with increasing pressure. Mo $\mathrm{K} \alpha$ irradiated.

The high pressure amorphous $\mathrm{GeO}_{2}$ taken out from DAC after decompression was immersed in methylene iodide saturated with sulphur and examined under a polarizing microscope. The fragment was optically isotropic and showed very high relief. The relief under the microscope depends upon the difference between the refractive index of the fragment and media. The degree of relief also depends on the numerical aperture of substage condenser. These relations can be applied to estimating the refractive index of fragment. ${ }^{11)}$ The value of refractive index of the high pressure amorphous $\mathrm{GeO}_{2}$ approximated by this method was greater than those of $\mathrm{q}-\mathrm{GeO}_{2}(\omega=1.695, \varepsilon=1.735)$ and less than those of $\mathrm{r}-\mathrm{GeO}_{2}(\omega=1.99, \varepsilon=2.05-2.10),{ }^{12)}$ although the precise value could not be obtained.

From the above optical study, we can assume that the quenched amorphous $\mathrm{GeO}_{2}$ is considered to have a higher density than $\mathrm{q}-\mathrm{GeO}_{2}$. The measurements of density of high pressure amorphous $\mathrm{GeO}_{2}$, which was synthesized by a multi anvil high pressure apparatus, proved this consideration. ${ }^{13)}$ The result is also consistent with the XANES experiment ${ }^{14)}$ which showed that the amorphization was accompanied by the increase of the coordination of Ge atoms from fourfold to sixfold.

In summary, the amorphization of $\mathrm{GeO}_{2}$ at about $6.5 \mathrm{GPa}$ proceeds quickly with the large volume change at room temperature without large thermal energy, although the transformation from $\mathrm{q}-\mathrm{GeO}_{2}$ to amorphous $\mathrm{GeO}_{2}$ is irreversible in spite of low activation energy. Since the present amorphization was completed within a very short time interval less than $5 \mathrm{~ms}$, the position of nucleation and the direction of the propagation of the transformed phase were not determined. The matrix phase (crystalline phase) was observed to have little structural change prior to the amorphization.

\section{References}

1) Mishima, O., Calvert, L. D., and Whalley, E. (1984): Nature, 310, 393-395.

2) Fujii, Y., Kowaka, M., and Onodera, A. (1985): J. Phys., C 18, 789-797.

3) Hemley, R. J. et al. (1988): Nature, 334, 52-54.

4) Kruger, M. B., and Jeanloz, R., (1990): Science, 249, 647-649. 
5) Williams., Q. et al. (1990): J. Geophys. Res., 95, 21549-21563.

6) Yamanaka, T. et al. (1992): High Pressure Research; Application to Earth and Planetary Science (eds. Y. Syono and M. H. Manghnani). Terra Scientific Publishing Company, Tokyo, Japan, pp. 493-501.

7) Finch, C. B., and Clark, G. W. (1968): Am. Mineralogist, 53, 1394-1398.

8) Yamaoka, S. et al. (1979): Rev. Sci. Instrum., 50, 1163-1164.

9) Piermarini, G. J., Block, S., and Barnett, J. D. (1973): J. Appl. Phys., 44, 5377-5382.

10) Forman, R. A. et al. (1972): Science, 176, 284-285.

11) Takubo, H., and Kataoka, H. (1990): J. Mineral. Soc. Japan., 15, $255-262$ (in Japanese).

12) Laubengayer, A. W., and Morton, D. S. (1932): J. Am. Chem. Soc., 54, 2303-2320.

13) Kawasaki, S., Ohtaka, O., and Yamanaka, T.: (in preparation).

14) Itie, J. P. et al. (1989): Phys. Rev. Lett., 63, 398-401. 